\section{過去の神経活動がどのように現在のスパイク頻度に影響を与 えるのか}

How past neuronal activity affects the current firing rate

Tomohiro Shirakawa, Hiroshi Sato (Dept. Comp. Sci., NDA)

The plasmodium of Physarum polycephalum is a unicellular and multinuclear giant amoeba. In the previous studies, we found some allometric scaling laws in the cell motility and exploratory behavior of the plasmodium. For example, the area of plasmodial body is proportional to the cell weight to the power of three fourths, and the velocity of cell motility is proportional to the length of the plasmodium. In this study, we tried to investigate how such allometries emerge, and found that the allometric laws can be explained in terms of the dynamics of cytoplasmic streaming.

2P279 Analysis for the exploratory behavior of Physarum plasmodium in an unlimitedly extendable space

Miharu Nishida, Hiroshi Satou, Tomohiro Shirakawa (Dept. Comp. Sci., $N D A)$

The plasmodium of Physarum polycephalum is a unicellular giant amoeba. Recent studies clarified that the plasmodium has an ability to perform computation such as maze-solving and optimization of a network. These studies targeted the behavior of the plasmodium in a closed space and thus there is no study on the long-term exploratory behavior of the organism in an open and wide space. In this study, we developed an experimental system that provides unlimitedly extendable 2-dimensional space. The setup consists of 9 square plates with wet paper towel substrate on each of them. By rearranging the plates according to the motility of the plasmodium, we can extend the 2-dimensional space arbitrarily. As a result, we succeeded to observe long-term motility of the plasmodium.

\section{Cell motility of the Physarum plasmodium on a non-uniform} substrate

Shinji Ishiguro, Hiroshi Sato, Tomohiro Shirakawa (National Defense Academy of Japan)

The plasmodium of true slime mold Physarum polycephalum is a unicellular and multinuclear giant amoeba. Since the plasmodium has amorphous body, the cell motility of the plasmodium is continuous and omnidirectional. This nature makes the motility of the plasmodium complex and gives rise to some difficulty in observation. Therefore we developed an experimental system that is able to limit the motility of the plasmodium. In our experiment, we used a non-uniform substrate such that the hydrophilic surfaces with circular shape are arranged in a 2dimensional lattice and surrounded by hydrophobic plastic surface. By using this substrate, we succeeded to limit the motility of the plasmodium to 4-directional neighbors, and to stepwise one.
Negative feedback regulation of KaiC ATPase gives origin to the circadian periodicity of cyanobacteria

Atsushi Mukaiyama ${ }^{1,2,3}$, Masato Osako ${ }^{4}$, Takaaki Hikima ${ }^{3}$, Takao Kondo ${ }^{4}$ Shuji Akiyama ${ }^{1,2,3}$ ( ${ }^{1}$ Inst. Mol. Sci., ${ }^{2}$ Grad. Univ. for Adv. Studies (SOKENDAI), ${ }^{3}$ SPring-8, RIKEN, ${ }^{4}$ Nagoya Univ.)

Circadian clocks are endogenous timing devices to accommodate the biochemical and physiological processes to dairy alterations in external environments. In cyanobacterium Synechococcus elongatus PCC7942, circadian clocks consist of the three proteins, KaiA, KaiB and KaiC. In vitro incubation of the three Kai proteins with ATP generates the phosphorylation cycle of KaiC with a period of approximately $24 \mathrm{~h}$.

The frequency of the phosphorylation cycle is linearly correlated with the ATPase activity of KaiC alone. This means that KaiC ATPase determines the period. Additionally, KaiC ATPase is extremely low, and temperaturecompensated, suggesting that it seems to be under negative feedback regulation. In this meeting, we provide experimental evidences to support

\section{マイクロドロップレットで構築された非平衡人工細胞の実験 的 - 数理的解析}

Experimental and numerical analyses of microdroplet-based nonequilibrium artificial cells

Masahiro Takinoue ${ }^{1,2}$, Haruka Sugiura ${ }^{1}$, Hiroyuki Kitahata ${ }^{3}$, Yoshihito Mori ${ }^{4}$ ( Interdisciplinary Grad. Sch. Sci. \& Eng., Tokyo Tech., ${ }^{2}$ PRESTO, JST, ${ }^{3}$ Dept. Phys., Chiba Univ., ${ }^{4}$ Dept. Chem., Ochanomizu Univ.)

Recently, construction of artificial cells as simplified models of living cells has been proposed to study essential dynamic mechanisms of life systems. However, most of them have limitations in transporting exterior/interior molecules through their interface, preventing implementation of sustained dynamic nonequilibrium reactions such as nonlinear oscillations. Here, we demonstrate an artificial cell construction based on water-in-oil microdroplets. In this system, the artificial cell was kept nonequilibrium by the controlled influx/efflux of molecules in a microfluidic channel; sustained nonlinear chemical oscillations were successfully achieved. We believe that this system will promote the study of dynamic chemical systems in artificial cells in future. 